\title{
La manipulation de la commutation de l'hémoglobine : approche thérapeutique des hémoglobinopathies
}

La thérapeutique de la drépanocytose, quarante ans après la mise en évidence du défaut moléculaire qui en est responsable, reste encore très largement un traitement symptomatique et le traitement des complications majeures : analgésiques, hydratation, transfusions dans certains cas, vaccinations, antibiotiques... Tous les essais de produits inhibiteurs de la polymérisation se sont en effet jusqu'à présent heurtés à l'impossibilité d'atteindre les concentrations pharmacologiques efficaces, ou à celle du passage de la barrière cellulaire, ou enfin à l'obstacle de leur toxicité par fixation sur des protéines autres que l'hémoglobine. Les produits modifiant l'équilibre hydrique et ionique sont mal tolérés et ceux qui agiraient sur la microvascularisation sont apparemment peu efficaces, sauf peut-être au niveau rétinien. Dans le but de mieux tester les composés pharmacologiques potentiels, on a cherché à réaliser un modèle murin de la maladie. Si plusieurs variantes de souris transgéniques existent actuellement, dont certaines font des drépanocytes, aucune cependant ne présente vraiment tous les troubles physiopathologiques de la maladie humaine, en particulier l'anémie et l'hémolyse ( $\mathrm{m} / \mathrm{s} n^{\circ} 3$, vol. $\left.6, p .314\right)$. $\mathrm{C}$ 'est dans une autre direction qu'ont été orientées ces dernières années tout un faisceau de recherches. Puisqu'il est établi que la polymérisation est liée au taux intracellulaire d'hémoglobine $\mathrm{S}$, et que d'autres hémoglobines qui ne copolymérisent pas, en particulier l'hémoglobine fœtale $(\mathrm{HbF})$, inhibent cette polymérisation, existe-t-il une approche pharmacologique permettant d'induire la synthèse de cette $\mathrm{HbF}$ ? Cette à un nombre limité de cellules, peuton également approcher plus ou moins d'une distribution pancellulaire ? Au cours de la 7th Conference on hemoglobin Switching qui s'est tenue à Airlie House du 8 au 11 septembre 1990, une séance a été consacrée à cette question de la manipulation in vivo de la commutation $\mathrm{HbF} \rightarrow \mathrm{HbA}$, normalement périnatale.

Dans une première approche (George Stamatoyannopoulos, Seattle, WA, États-Unis) ont été passés en revue les stimuli susceptibles d'induire l'HbF chez l'adulte, le mécanisme pouvant être soit la production de novo de facteurs agissant en trans dans des cellules en régénération rapide, soit la maturation accélérée d'érythroblastes contenant des facteurs "fotaux ". Dans un cas comme dans l'autre, il pourrait s'agir d'une régulation positive ou d'une régulation négative, stimulation ou inhibition. Les agents pharmacologiques susceptibles d'induire la synthèse d'HbF peuvent être rangés en trois catégories :

1) ceux qui agissent sur le cycle cellulaire et les cytotoxiques, parmi lesquels il faut citer la 5-Aza-Cytidine (5-Aza-C), l'hydroxyurée (HU), la cytarabine (Ara $\mathrm{C}$ ), la vinblastine, le méthotrexate ;

2) certaines cytokines, au premier rang desquels l'érythropoïetine (Epo) mais aussi une interleukine, l'II-3 ; 3) un troisième groupe enfin comporte l'acide butyrique et ses dérivés. Des synergies ont aussi été démontrées, entre butyrate et $5-\mathrm{Aza}-\mathrm{C}$, de même qu'entre $\mathrm{HU}$ et Epo.

Des essais thérapeutiques ont par ailleurs été présentés. Un essai pluricentrique (George Dover, Johns Hopkins
Hosp., Baltimore, MD, États-Unis) a mesuré les effets d'un traitement par l'HU dans la drépanocytose. Les meilleurs résultats ont été obtenus par administration quotidienne d'une dose qu'on augmente progressivement jusqu'à la limite de la toxicité, variable pour chaque individu. L'augmentation statistique de l' $\mathrm{HbF}$ et des réticulocytes $\mathrm{F}$ est importante, mais variable, avec des sujets " répondeurs" et des sujets " nonrépondeurs", sans que l'on puisse jusqu'à présent cerner les paramètres génétiques responsables de cette réponse. Il faut noter qu'il y a augmentation non seulement de l'HbF, mais aussi de l'HbS; globalement cependant, il y a diminution de la polymérisation, d'où diminution de la densité et augmentation de l'élasticité cellulaires. Aucun effet myélotoxique secondaire significatif n'a été observé. La réduction de fréquence des crises vaso-occlusives semble de l'ordre de $50 \%$. Sous réserve de contrôles très systématiques, les essais cliniques devraient donc se poursuivre.

Un autre essai (Griffin Rodgers, NIH, Bethesda, MD, États-Unis) a porté sur un nombre plus limité de sujet et la mise au point d'un protocole de traitement associant l'HU et l'Epo recombinante. La base du traitement est ici l'administration d'HU quatre jours de suite chaque semaine, et l'administration d'Epo les jours 5 à 7. L'HU, comme dans l'essai précédent, détermine une augmentation de l'HbF et des réticulocytes $\mathrm{F}$, les taux obtenus se stabilisant à un plateau. L'adjonction d'Epo à ce traitement de base détermine une augmentation immédiate des deux paramètres (HbF jusqu'à 15-20 \% ; réticulocytes $F$ jusqu'à 40-45 \%) ainsi 
qu'une amélioration de la filtrabilité des cellules.

Un autre essai (Ronald Nagel, Albert Einstein, Bronx, NY, Etats-Unis) concernait un traitement par l'Epo seule à différentes doses, mené en double aveugle. Les résultats, quoique incontestables, sont ici plus modestes.

Enfin une gamme d'associations variées a été essayée chez le babouin (K. Mac Donagh, NIH, Bethesda, MD, États-Unis), animal de choix car sa ligne de base de cellules $\mathrm{F}$ est faible.

On a ainsi essayé :

- L'HU quotidienne et le butyrate de $\mathrm{Na}$ discontinu. Chaque période de ce dernier traitement se traduit par un pic d'HbF et de réticulocytes $\mathrm{F}$ alors que les réticulocytes totaux diminuent.

- L'HU et l'Epo à divers rythmes. Là aussi, l'augmentation, ou la dimi-

'nution de l'HbF semble liée à l'addition d'Epo au traitement de base.

- L'HU et l'II-3 semblent provoquer peu de réponse. Il y a donc combinaison efficace dans les deux premiers cas. Le butyrate semblerait agir en augmentant la réponse à l'HU.

Deux communications enfin concernaient spécifiquement l'action du butyrate. Sur un modèle ovin (Suzan
Perrine, Oakland, CA, États-Unis) on a montré qu'on peut retarder chez la brebis la commutation périnatale de l'hémoglobine qui est analogue à celle de l'homme. Des recherches sont en cours pour trouver le dérivé synthétique stable qui permettrait une inhibition permanente ou même une inversion de la commutation. Les mécanismes responsables de cette inhibition ont été évoqués : rapport $\gamma / \beta$, taux d'AMP cyclique, acétylation des histones, différenciation de la morphologie érythrocytaire, action au niveau des promoteurs $\gamma$ et $\beta$.

Enfin, dans un modèle aviaire, l'inversion de commutation a été étudiée chez le poulet (G. Ginger, Minneapolis, MN, États-Unis). La réactivation du gène embryonnaire $R$ ho est obtenue par l'association de la 5-Aza-C, agent déméthylant de l'ADN, et l'action stimulatrice du butyrate, alors que la même action synergique n'est pas observée quand on associe les autres cytotoxiques au butyrate.

Bien que fragmentaires, ces résultats sont donc positifs et semblent enfin permettre d'entrevoir une voie thérapeutique nouvelle de la drépanocytose, et même des thalassémies.

D.L.

\section{ERRATA}

- Il était indiqué dans le flash sur l'inversion de la sénescence par un anti-messager $\left(\mathrm{m} / \mathrm{s} n^{\circ} 8, \mathrm{vol}\right.$. 6, octobre 1990, page $811)$ que la sénescence de Podospora auscrina était liée à une amplification incontrôlée d'un ADN mitochondrial tronqué. II fallait lire, bien entendu, Podospora anserina.

- Dans le dernier numéro de $\mathrm{m} / \mathrm{s}$ ( $n^{\circ} 8$, vol. 6, octobre 1990), le nom de Christine Petit (co-auteur avec Jean Weissenbach de l'article intitulé : "Chromosome $Y$ et détermination du sexe ") ne figurait pas en page de couverture et dans le sommaire. Nous prions l'auteur de bien vouloir nous en excuser.

\section{1] BRÈVE}

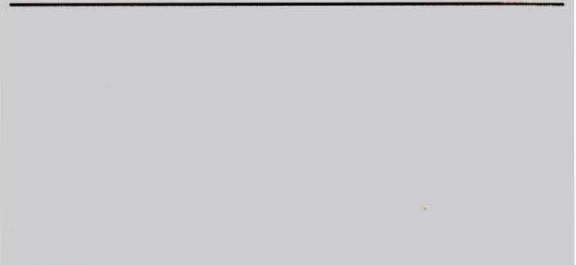

पa L mutation scid de la souris est-elle une nouvelle réparatose ? La mutation Scid (severe combined immunodeficiency) de la souris est caractérisée, comme son nom l'indique, par un déficit immunitaire grave intéressant tout à la fois les lignées de lymphocytes $B$ et $T$. La base moléculaire de ce déficit est un réarrangement aberrant, non productif, des segments de gène codant pour les immunoglobulines et les récepteurs de lymphocytes (TcR). Ces anomalies avaient conduit à proposer que le déficit était lié à une altération primaire du système enzymatique de la recombinase, responsable du réarrangement aussi bien des gènes des immunoglobulines que des $\mathrm{TcR}$ au cours de la différenciation lymphocytaire. En fait, de récents résultats d'une équipe canadienne de Toronto indiquent qu'existe, chez les souris scid, un déficit généralisé de la réparation de l'ADN, marqué par une sensibilité accrue aux radiations ionisantes de toutes les cellules testées, notamment les cellules myéloïdes et les fibroblastes [1]. Ces résultats sont cohérents avec la localisation du gène scid sur un autre chromosome que celui portant les gènes $R A G-1$ et $R A G$-2 dont on suppose qu'ils pourraient coder pour des sous-unités de la recombinase $\left(\mathrm{m} / \mathrm{s} n^{\circ} 8\right.$, vol. 6 , p. 820); ils indiquent aussi que des systèmes normalement impliqués dans la réparation ubiquitaire de l'ADN interviennent également dans le réarrangement spécifique des gènes d'immunoglobuline et de $T c R$. Ce fait pourrait expliquer la fréquence des atteintes immunitaires, quoique bien moins sévères que chez la souris scid, observées au cours des maladies de la réparation de l'ADN (les réparatoses). D'un point de vue pratique, l'information que l'expression du gène scid est ubiquitaire facilitera sûrement son clonage moléculaire. [1. Fulop GM, Phillips RA. Nature $1990 ; 347$ : 479-82.
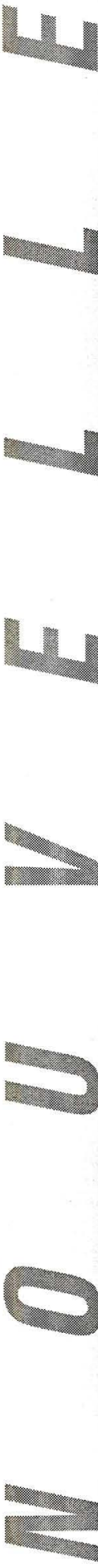